\title{
Grain breakage under uniaxial compression, through 3D DEM modelling
}

\author{
François Nader $^{1, \star}$, Claire Silvani ${ }^{1, \star \star}$, and Irini Djeran-Maigre ${ }^{1, \star \star \star}$ \\ ${ }^{1}$ Univ Lyon, INSA-Lyon, SMS-ID, F-69621, France
}

\begin{abstract}
A breakable grain model is presented, using the concept of particles assembly. Grains of polyhedral shapes are generated, formed by joining together tetrahedral subgrains using cohesive bonds. Single grain crushing simulations are performed for multiple values of the intra-granular cohesion to study the effect on the grain's strength. The same effect of intra-granular cohesion is studied under oedometric compression on samples of around 800 grains, which allows the evaluation of grain breakage model on the macroscopic behaviour. Grain size distribution curves and grain breakage ratios are monitored throughout the simulations.
\end{abstract}

\section{Introduction}

Rockfill structures are becoming more common in civil engineering, mainly for the benefits they present on a practical and economical scales. These structures are subjected to loads that can lead to grain breakage, which in turn modifies the grain size distribution of the material, and therefore its behaviour.

To simulate the behaviour of this type of material, the discrete element method is usually chosen for its ability to capture the discrete nature characteristic of grains. Many breakage models are suggested in the literature that can be grouped into two principal concepts. The first concept is based on replacing a grain with a number of smaller grains when an induced stress threshold is reached. This concept was used by Ästrom and Hermann 1998 [1], Tsoungui 1999 [2], and Ben-Nun and Einav 2010 [3] who modelled grains as discs. McDowell and De Bono 2013 [4] used the same concept with three-dimensional spherical grains. Cantor et al. 2014 [5] modelled a grain as a rigid polygon that split in the direction of the principal stress.

The second concept consists of assembling subgrains using cohesive bonds to form a grain. The breakage of a grain is the consequence of the breakage of the cohesive bonds. Different types of subgrains have been studied in the literature. Silvani et al. 2009 [6] applied a MohrCoulomb cohesive law between rigid discs to form circular grains and simulated oedometric tests. Cheng et al. 2003 [7] applied cohesive bonds between small spheres to form spherical grains, removing $20 \%$ of the subgrains to induce defects inside the grain and reduce its strength. Nguyen et al. 2015 [8] used a two-dimensional Voronoï tessellation to divide a circular grain into smaller polygonal particles and joined them together using cohesive bonds. Cantor et al. [9] 2016 used a three-dimensional Voronoï tessellation

\footnotetext{
^ e-mail: francois.nader@insa-lyon.fr

$\star \star$ e-mail: claire.silvani@insa-lyon.fr

$\star \star \star$ e-mail: irini.djeran-maigre@insa-lyon.fr
}

to divide a spherical grain into polyhedral particles joined together by a cohesive Tresca law.

In the work presented hereafter, the second concept is adopted, which consists of assembling rigid tetrahedral subgrains using a Mohr-Coulomb cohesive law to generate three-dimensional polyhedral grains. The grain generation algorithm is described, single crushing and oedometric compression simulations are presented for different values of the intra-granular cohesion. The goal of the study is to create a model in which the angularity of grains is taken into account, an aspect that cannot be studied when circular or spherical grains are used, and to show the effect of grain breakage and intra-granular cohesion on the macroscopic behaviour.

\section{Grain generation}

Grain shape plays a major role in the distribution of contact forces inside granular media, and in the overall macroscopic behaviour. To take this into account, polyhedral grains are generated. The generation algorithm takes in input the maximum grain dimension $2 r_{\text {max }}$, the elongation $e=\frac{r_{\max }}{r_{\min }}$ where $2 r_{\min }$ is the minimum grain dimension, the number of half-planes $N_{h p}$ and the number of vertices $N_{v}$ in each half-plane. The center point of the polyhedron is placed along with the top and bottom vertices. Then in a vertical half-plane, $N_{v}$ vertices are placed at a random distance $r$ from the center, such as $r_{\text {min }}<r<r_{\text {max }}$ (figure 1.a). The second half plane is then selected by rotating around the vertical axis, and the same process is repeated until the $N_{h p}$ half-planes are filled. The vertices are joined to form the polyhedron. Figure 1.b shows a sample of 30 randomly generated polyhedra. The meshing software Gmsh is used to divide the polyhedron into elementary tetrahedra, that are then joined together using a Mohr-Coulomb law to form the breakable polyhedral grain. 


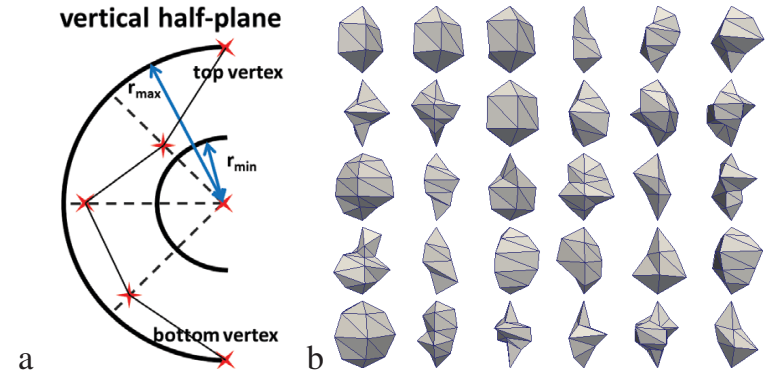

Figure 1. a. Grain generation - b. Sample of randomly generated polyhedra

\section{Single grain crushing}

Single grain crushing simulations are carried out to evaluate the grain's strength for different values of the intra-granular cohesion.

The open-source software $L M G C^{90}$ is chosen to run the simulations. This software implements the discrete element method called Non-Smooth Contact Dynamics (NSCD) [10, 11]. This method takes into consideration the dynamics of each element, as well as the interactions between the bodies, in order to describe the overall behaviour of the medium.

A breakable polyhedral grain is placed between two rigid plates. An increasing downward vertical load is applied on the upper plate until the grain breaks (figure 2). The simulated grain has a maximum size of $4 \mathrm{~cm}$ divided into 12 subgrains. Multiple values of the intra-granular cohesion are tested, varying from $1 \mathrm{kPa}$ to $10^{4} \mathrm{kPa}$. The grain's reaction on the upper plate increases and reaches a peak value that is considered equal to the grain's breakage force. Figure 3 shows the evolution of the grain's breakage force as a function of the cohesion. Each point on the curve represents the average breakage force of a set of 15 single crushing simulations, run on grains having a similar size, the same order of magnitude of subgrain size, but different number of vertices. Error bars represent standard deviation.

The breakage force increases from $13 N$ to $1500 N$. This increase can be explained by the fact that when the value of the intra-granular cohesion increases, the force needed to break the bond increases, which in turn leads to an increase in the breakage force.

\section{Oedometric simulations on a multigrain sample}

A series of oedometric compression simulations are conducted on a sample of 855 grains, deposited by gravity in a rectangular box of a horizontal section of $30 \mathrm{~cm} \times 30 \mathrm{~cm}$, and a height of $26 \mathrm{~cm}$. All the grains have a maximal grain size of $4 \mathrm{~cm}$, with a number of vertices varying randomly between 8 and 14. All grains have an elongation equal to 1.0 (regular polyhedra), and each grain is divided into 8 to 12 tetrahedral subgrains (figure 4). Gravity is not taken

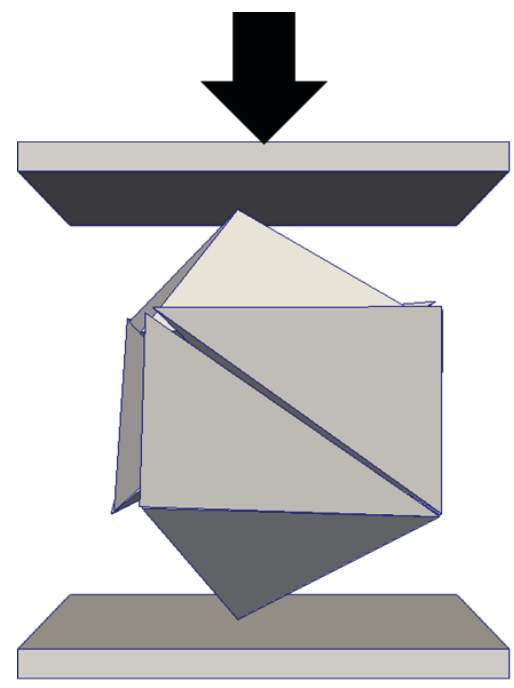

Figure 2. Single grain crushing under vertical load

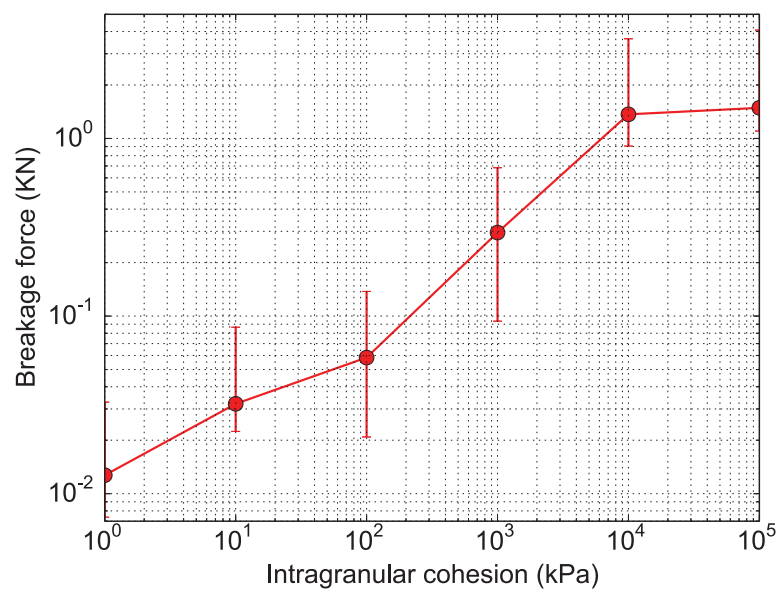

Figure 3. Breakage force as a function of the intra-granular cohesion

into account during compression.

Figure 5 shows the evolution of the void ratio as a function of the vertical stress, on a semi-logarithmic scale, for three values of the intra-granular cohesion: $1 \mathrm{kPa}, 10^{2} \mathrm{kPa}$ and $10^{4} \mathrm{kPa}$. Many grain/plate contacts appear and disappear during compression, generating noise in the curves involving the vertical stress, the void ratio and the vertical strain. To cancel the noise and have clearer curves, the median value of these parameters is calculated over an interval of numerical steps. A slight decrease in the void ratio is observed at the beginning until the applied stress reaches $10 \mathrm{kPa}$, where the slope becomes steeper indicating a more important decrease in the void ratio with the increasing vertical stress. The difference between the three simulations is not clearly noticeable on these curves, therefore additional parameters are explored. Figure 6 shows the evolution of the vertical stress as a function of the vertical strain. The median values are shown in this figure. The effect of the cohesion is more visible: for a stronger cohesion, a bigger vertical stress is needed to obtain the same 
vertical strain, which means that a higher intra-granular cohesion reduces the compressibility of the sample. The effect of the intra-granular cohesion on the macroscopic behaviour of the studied material could be clearer. This can be a result of the boundary conditions imposed by the oedometric compression. this can also explain why the vertical strain strain reached in the oedometric compression does not show the decrease in the compressibility usually seen in this type of material.

To further analyse the behaviour, the percentage of the remaining cohesive bonds is monitored during compression (figure 7). It is clear that grains that have a lower intragranular cohesion break for low values of vertical strain, therefore under lower loadings, which is in agreement with the observations made in section 3 .

The evolution of the grain size distribution is monitored throughout the simulations. Figure 8 shows a typical evolution of the grain size distribution (in this case for a cohesion of $10^{4} \mathrm{kPa}$ ) for multiple vertical strains. Due to the uniform initial granular size distribution, as well as the rigid nature of the model, the maximal grain size decreases during compression as can be seen when comparing the successive grain size distribution curves. The final state curve is defined as the state where all cohesive bonds are broken, so the elementary tetrahedral subgrains become grains.

To quantify this evolution of the grain size distribution, the breakage ratio is computed at multiple vertical stresses, using the formula proposed by Einav 2007 [12] :

$$
B_{r}=\frac{B_{t}}{B_{p}}
$$

where $B_{r}$ is the breakage ratio, $B_{t}$ is the area between the initial and the current distribution, and $B_{p}$ is the area between the initial and the final distribution. Einav 2007 [12] suggests the use of a fractal distribution $F(d)$ for the final state $F(d)=\left(\frac{d}{d_{M}}\right)^{3-D}$ where $d$ is the grain size, $d_{M}$ the maximum grain size and $D$ the fractal dimension. Instead, the final distribution where all the cohesive bonds are broken is chosen because it is indicative of the limitation of the numerical model, since it represents the last state the simulated sample of grains can reach. Figure 9 shows the evolution of this breakage ratio as a function of the intra-granular cohesion, for two values of the vertical stress. Lower values of the cohesion show higher breakage ratios, which means that more grains are broken for the same value of the vertical stress. This result is also in agreement with the previous conclusions: stronger cohesions lead to less grain breakage under the same applied stress.

\section{Conclusion}

The results of this study can be summarised by the following conclusions :

A grain generation algorithm is created. It allows the generation of complex polyhedral shapes of grains. A meshing of the generated polyhedron is used to turn it into a breakable grain.

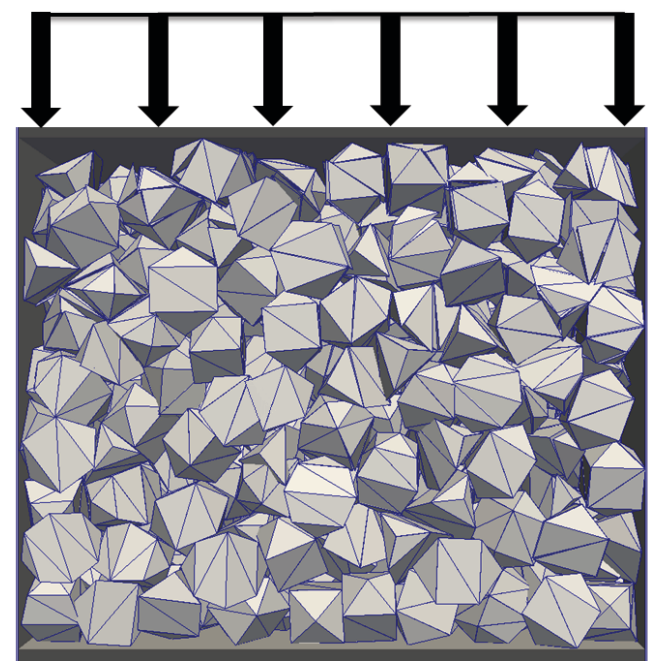

Figure 4. Sample of breakable grains during the oedometric compression

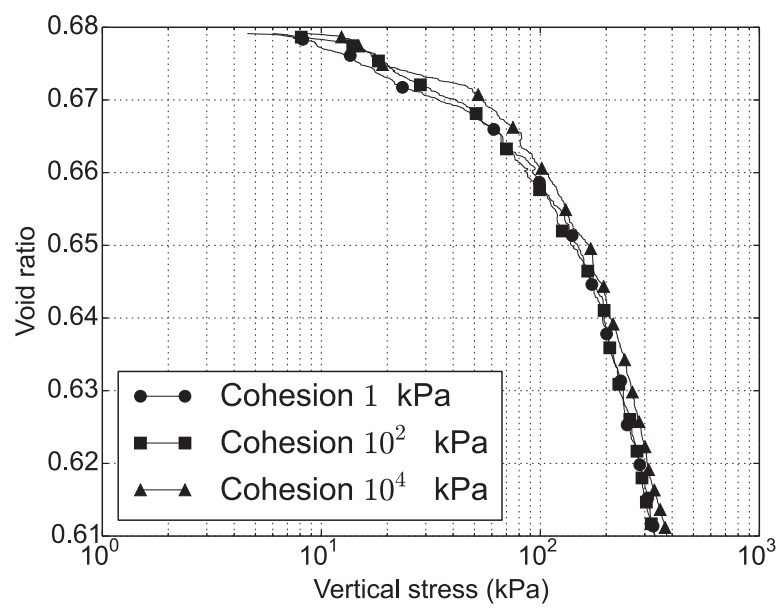

Figure 5. Oedometric curves for for three different values of the intra-granular cohesion (median values)

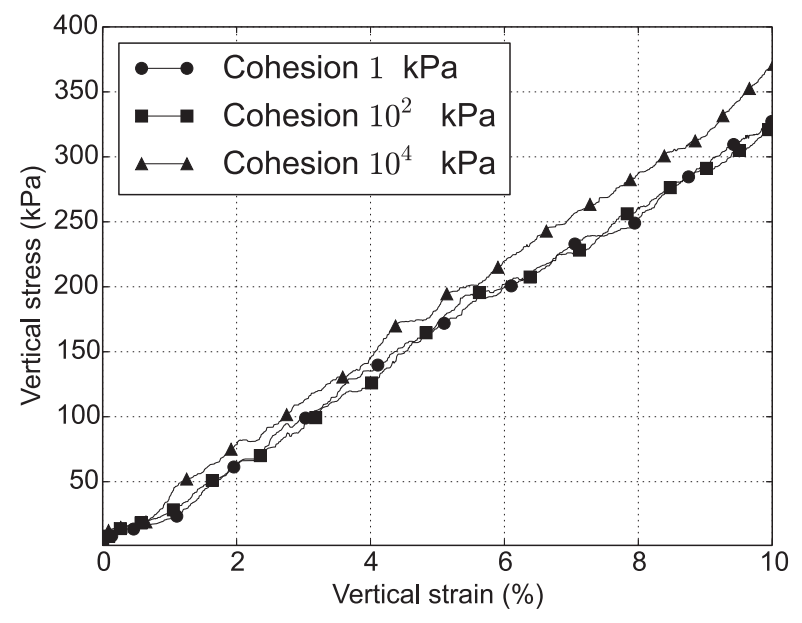

Figure 6. Vertical stress as a function of vertical strain for for three different values of the intra-granular cohesion (median values) 


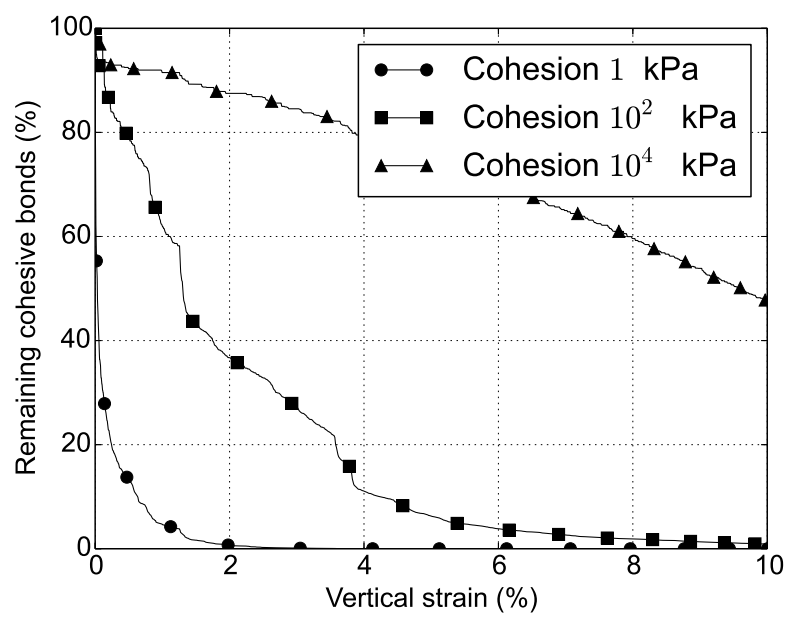

Figure 7. Remaining cohesive bonds as a function of vertical strain for three different values of the intra-granular cohesion

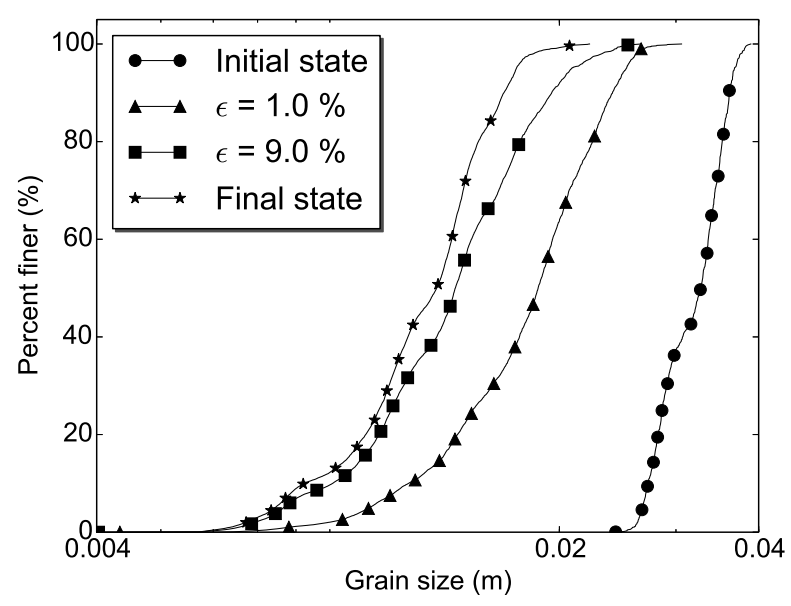

Figure 8. Grain size distribution throughout an oedometric compression

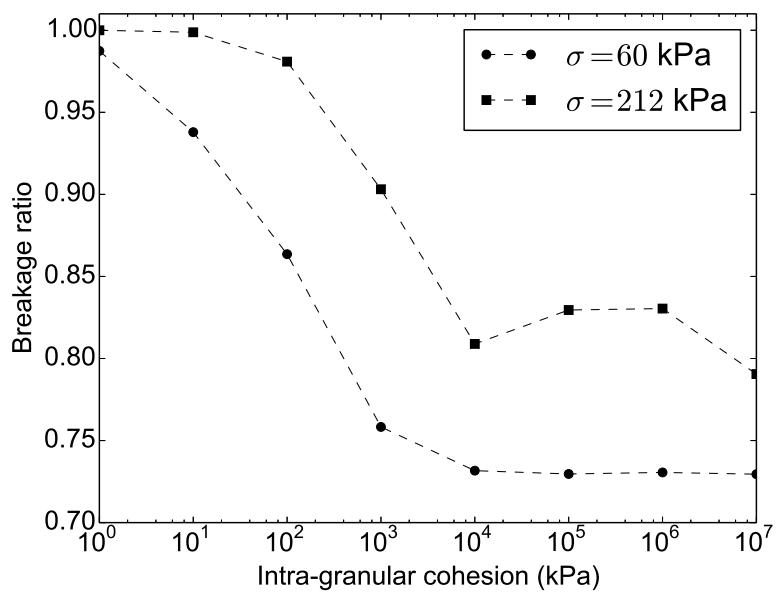

Figure 9. Breakage ratio as a function of intra-granular cohesion
Regular polyhedra are selected to conduct the single grain crushing and the oedometric compression simulations.

A single grain's strength depends on the intra-granular cohesion joining its subgrains: the higher the cohesion, the stronger the grain.

The oedometric simulations also show the effect of intragranular cohesion: the higher the cohesion, the lower the compressibility of the sample. Nevertheless, this effect is limited as a result of the boundary conditions imposed by the oedometric conditions, the ratio between the oedometric cell size and the grain size, as well as the meshing of the grain (its division into subgrains). Triaxial simulations might allow additional rearrangement of grains, thus leading to different macroscopic behaviour. Other potential solutions are increasing the size of the oedometric cell or refining the grain meshing. On the microscopic scale, the effect of the intra-granular cohesion is clearer. A higher applied stress is needed to break grains that have a higher intra-granular cohesion. These observations, along with the evolution of the grain size distribution throughout the compression, show that this numerical model, that joins subgrains using cohesive bonds, manages to simulate grain breakage.

Further work is planned to study the effect of the meshing and a broader initial granular size distribution on the macroscopic behaviour.

\section{References}

[1] J. Åström, H. Herrmann, Eur. Phys. J. B 5, 551 (1998)

[2] O. Tsoungui, D. Vallet, J. Charmet, Powder Technol. 105, 190 (1999)

[3] O. Ben-Nun, I. Einav, Philos. Trans. R. Soc. London, Ser. A 368, 231 (2010)

[4] G. McDowell, J. De Bono, Géotechnique 63 (2013)

[5] D. Cantor, N. Estrada, E. Azéma, Comput. Geotech. 67, $150(2015)$

[6] C. Silvani, T. Désoyer, S. Bonelli, Int. J. Numer. Anal. Methods Geomech. 33, 665 (2009)

[7] Y. Cheng, Y. Nakata, M. Bolton, Géotechnique 53, $633(2003)$

[8] D.H. Nguyen, P. Sornay, E. Azéma, F. Radjai, Geomechanics from Micro to Macro 1, 275 (2014)

[9] D. Cantor, E. Azéma, P. Sornay, F. Radjaï, Comput. Part. Mech. pp. 1-10 (2016)

[10] J.J. Moreau, in Nonsmooth Mechanics and Applications (Springer, Vienna, 1988), pp. 1-82

[11] M. Jean, Comput. Methods Appl. Mech. Eng. 177, 235 (1999)

[12] I. Einav, J. Mech. Phys. Solids 55, 1274 (2007) 